\title{
Lidil
}

Revue de linguistique et de didactique des langues

La réception des textes littéraires

\section{La poésie contemporaine}

L'institution scolaire et les " règles de l'art "

Pierre Ceysson

\section{(2) OpenEdition}

Journals

Édition électronique

URL : http://journals.openedition.org/lidil/78

DOI : $10.4000 /$ lidil.78

ISSN : 1960-6052

Éditeur

UGA Éditions/Université Grenoble Alpes

Édition imprimée

Date de publication : 1 juin 2006

Pagination : 37-54

ISBN : 2-914176-14-7

ISSN : 1146-6480

Référence électronique

Pierre Ceysson, «La poésie contemporaine », Lidil [En ligne], 33 | 2006, mis en ligne le 01 décembre 2007, consulté le 19 avril 2019. URL : http://journals.openedition.org/lidil/78 ; DOI : 10.4000/lidil.78

Ce document a été généré automatiquement le 19 avril 2019

(C) Lidil 


\title{
La poésie contemporaine
}

\author{
L'institution scolaire et les « règles de l'art »
}

\author{
Pierre Ceysson
}

1 Nouveau, moderne, avant-garde, contemporain, extrême contemporain, autant de termes que l'on peut nominaliser ou joindre à roman, poésie, poétique. Depuis longtemps, le neuf se constitue toujours avec et contre de l'ancien'. Il faut s'en souvenir à un moment où l'institution culturelle et l'institution scolaire envisagent et mettent en place des projets et des dispositifs novateurs, et il m'a paru utile d'interroger la situation actuelle de la poésie dans les différents lieux de sa production, de sa promotion et de sa consommation. Il doit bien y avoir quelques règles de l'art (Bourdieu, 1992) à l'œuvre dans l'institution scolaire, dans le micro-champ poétique et aux intersections de l'une et de l'autre. Examinons «du haut jusques en bas»: du recueil au manuel scolaire, des savants aux néophytes, des éminents aux médiateurs...

«Absolument moderne » : autour de l'opposition « l'écriture »/ « habiter en poète »

2 S'il prend parti dans le cadrage de la nouvelle poésie française et établit un palmarès inévitablement discutable et discuté, Le Magazine littéraire propose aussi une problématisation de ce qu'est le contemporain et de ce qu'est le moderne. La poésie depuis 1970 est globalement orientée par une double postulation ${ }^{2}$, assurément relayée par des enjeux et des prises de position opposés :

3 - d'une part, une poésie caractérisée par un travail sur le signifiant comprend ce qui relève du textualisme et de la littéralité (de Tel Quel à Jean-Marie Gleize en passant par Francis Ponge) ;

4 - d'autre part, une poésie de l'«habiter en poète", caractérisée soit par un souci ontologique soit par l'inscription dans « la circonstance », réunit les poètes de la présence au monde (Yves Bonnefoy) et le lyrisme critique le plus récent (Maulpoix, Goffette).

5 Terme fourre-tout qui, pour distinguer, légitimer ou classifier, a besoin de se nuancer (la catégorie de l'extrême contemporain en est l'exemple), la notion de contemporain est une "synthèse sans concept» (Meschonnic) susceptible de regrouper des pratiques et des esthétiques hétérogènes (Maurice Carême est bien contemporain d'Aragon, d'Eluard, de Ponge). Pour caractériser ce qui se définit comme écriture poétique aujourd'hui, la notion 
de modernité, qui relève " d'une généalogie, d'une historicité, d'une réflexivité ${ }^{3}$ ", est théoriquement plus satisfaisante, mais nécessairement polémique dans le discours des universitaires-poètes. Ainsi, l'actuelle modernité peut aller jusqu'à se définir contre une poétique du sujet et se revendique comme "poétique de l'évènement ", c'est-à-dire " rupture de la figuration et de la corporalisation ", que manifestent par exemple les cutup d'Olivier Cadiot ou la « langue saccadée » de Matthieu Messagier ; plus prudent quand il s'adresse aux bibliothécaires, Jean-Marie Gleize (2001) estime que la modernité est «traversée du monde par l'écriture d'un poète " - en cela, elle est historique et transhistorique (Ponge se rapproche de Rimbaud ou de Malherbe, et réciproquement) -, mais il ajoute que les néo-lyriques envisagent la modernité «sous un angle moins réformiste $"$.

Deux conséquences de ce positionnement. D'abord, le palmarès du Magazine littéraire enregistre les «nouvelles écritures [qui] s'appellent encore poésies » et retient quelques figures majeures, de poètes nés dans les années vingt, comme "devanci[ères] » de l'actuelle poésie: Bonnefoy, Du Bouchet, Dupin, Jaccottet. Sont exclus tous les poètes publiés dans des collections pour l'enfance et la jeunesse: si Cheyne éditeur est mentionné et si La Clarisse de Philippe Dumortier figure dans les publications récentes de la notice de l'éditeur, rien n'est dit sur la collection Poèmes pour grandir, pourtant pourvoyeuse essentielle des recettes de la maison; sont exclus des éditeurs importants, par exemple Le Dé bleu ou Actes Sud. Seconde conséquence: on se questionne sur la rythmique, le vers, la métaphore. Ainsi, quand il analyse le choix des proses en poésie, Gleize distingue d'une part une spécificité formelle, marquée dans le poème en prose, le chant du vers libre ou du verset, qu'il soit « de haut voltage (Pierre Oster) ou de basse tension (James Sacré) », d'autre part une prose «hors de la poésie », dont Ponge serait l'origine exemplaire (proèmes, définitions-descriptions...).

7 Comment les différentes strates de l'institution scolaire, non universitaire, se repèrentelles dans le contemporain et la modernité ?

Moderne/contemporain : palmarès scolairesPanachages poétiques : compromis et fourretout

Considérons le palmarès et les orientations des textes officiels. ${ }^{4}$ Dans les listes d'œuvres de littérature pour la jeunesse en principe destinées à la littérature cursive, les programmes du premier cycle pour les classes de sixième et du cycle central proposent des poètes publiés dans les collections pour la jeunesse (cinq poètes de la collection Poèmes pour grandir chez Cheyne au cycle central seulement ; cinq poètes de la collection Fleurs d'encre dirigée par Jacques Charpentreau - Pierre Coran, Maurice Carême, Georges Jean), des anthologies thématiques dont les Poèmes pour mes amis les enfants de Soupault, Les Animaux de Roubaud, Hugo, Prévert, Queneau, Claude Roy, les Innocentines d'Obaldia... Pour le cycle central, est effectuée la distinction entre les poètes contemporains modernes et ceux d'inspiration lyrique (on associe Philippe Jaccottet, Bernard Noël, Jean Tardieu, Frank Venaille avec Marc Alyn, René-Guy Cadou, Andrée Chédid, Jean-Hugues Malineau, Gianni Rodari), les poètes classiques (dans l'ordre, Hugo, La Fontaine, Marot, Rimbaud, Rutebeuf, Verlaine et Villon) et les poètes mis en chanson... En classe de troisième, les grands classiques (de Rutebeuf à Apollinaire) lisibles en anthologie sont accompagnés par un panachage d'auteurs contemporains réunissant des recueils de poètes de la fin du xixe siècle ou du début du xxe (Laforgue, Cros, Fargue), du xxe siècle (Aragon, Eluard, Prévert, Michaux, Prévert, Ponge, Queneau, Tardieu...) ; on s'étonne de trouver associés Le Visage de l'ange de Jacques Charpentreau, l'Anthologie personnelle de 
Frédéric-Jacques Temple, plus encore Tout le monde se ressemble d'Emmanuel Hocquard. Sans doute faut-il voir là des compromis établis avec des médiateurs de collections, de revues ou de commissions ministérielles, après l'enquête « lancée auprès des organismes spécialisés et des revues de littérature pour la jeunesse ». Le terme contemporain est bien un fourre-tout, mais la poésie pour la jeunesse est alors évacuée.

Sensibiliser au contenu et aux figures poétiques, puis mettre la poésie en séquence

S'il est conseillé d'approcher la poésie "pour le langage et les jeux» avec la préoccupation de regarder, lire, dire, écouter (classe de sixième); de "sensibiliser les élèves aux liaisons entre l'expression sonore et le contenu figuratif de la poésie", d'« identifier les figures-reines du discours et les symboles » et de proposer des exercices créatifs (cycle central), en classe de troisième, c'est dans le cadre d'une progression de séquence que s'intègre la poésie: on suggère d'intégrer un ensemble de textes de poètes de la Résistance (Aragon, Desnos, Eluard) dans une étude des formes d'argumentation, ou d'étudier l'engagement (forme de l'expression de soi) à partir de textes des mêmes auteurs, avec comme axe d'étude la poésie en lutte. Primauté du thématisme, quasi exclusivité des formes versifiées et rythmées, étude des figures du discours, polarisation en fonction des âges vers le ludique ou la poésie argumentative, autant de constantes qu'amplifient à peu de variantes près les douze manuels dépouillés 5 . On note l'exception dans l'étude des Enfantasques de Claude Roy (Besson, 2000 : 212-213): en cinq séances, servent de supports des questionnements sur les relations du texte et des images, la titrologie, le détournement des expressions figurées prises au pied de la lettre, les jeux homophoniques, la métrique et la structure strophique, l'énonciation, le procédé du collage ; on propose une réflexion terminale sur le contenu du livre ; l'objectif majeur est la réalisation d'un montage poétique au cours duquel les enfants en duo et par groupes diront les poèmes appris par cœur. La technique du collage n'est ni abordée par des jeux créatifs (mots-valises ou dessins), ni reliée à la fonction subversive du procédé (le refus du sérieux, la réfection de la fable traditionnelle, le détournement de la langue par une pratique du non-sens).

10 Le palmarès des auteurs contemporains s'établit comme suit dans l'ordre décroissant: Prévert, Apollinaire, Eluard, Roy, Guillevic ; les auteurs pour la jeunesse ne passent pas le niveau de la classe de cinquième ; parmi les contemporains considérés dans la modernité, seul Ponge apparait.

Le contemporain en médiations didactiques et pédagogiquesLégitimation de quelquesuns : rôle de la formation universitaire et pédagogique

11 Le cas de Ponge permet de faire apparaitre d'autres médiations dans la diffusion de la modernité, qu'il s'agisse de celle du lyrisme de l'habitation poétique du monde ou de celle du signifiant, dont Ponge est le représentant très majoritaire au collège et au lycée. Il n'est pas étonnant que le texte de L'huitre soit le plus fréquemment proposé ' $:$ les Entretiens Ponge/Sollers, l'étude de Gleize dans Poésie et figuration, le Ponge de la collection Textes pour aujourd'hui, le relais récent du Ponge de Serge Martin apportent un fondement critique suffisant pour un auteur déjà scolarisé depuis le milieu des années soixante-dix ( Tel Quel à l'université, Le Français aujourd'hui). Tout aussi importants pour la légitimation pédagogique apparaissent dans la formation des professeurs de Lettres les Onze études sur la poésie moderne de Jean-Pierre Richard, les publications des universitaires; dans l'information pédagogique, la revue l'école des lettres (celle du lycée plus que du collège), et plus particulièrement ses numéros spéciaux. C'est la raison majeure de la légitimation 
dans le second cycle plus particulièrement, de Ponge, mais aussi de Jaccottet, Bonnefoy, Char, Roubaud et l'Oulipo, exceptionnellement Guillevic, Dupin, du Bouchet '

Revues didactiques

C'est le rôle des revues de didactique de théoriser et de vulgariser l'ensemble de la poésie contemporaine: on fait alors de la transposition didactique. Si Le Français aujourd'hui préfère poser la question «Lisez-vous les contemporains?", Pratiques essaie de faire le point sur ce que signifie aujourd'hui « enseigner la poésie moderne ». Ainsi, devant le fait que la poésie n'existe plus ou « commence à échapper à toute définition, tout lieu, toute question d'origine ou d'inscription ", quand certains didacticiens renvoient le texte poétique à l'inclassable et ne proposent que l'apprentissage du commentaire composé ${ }^{\text {, }}$ on rappelle qu'enseigner la poésie implique le recours à des savoirs sociaux, techniques et historiques, que l'illisible est ce que « nos habitudes culturelles de lecture ne nous ont pas préparés/habitués à lire ». L'ambition de construire progressivement l'intelligibilité du poétique est considérable, qui vise à articuler la théorie, l'information la plus complète, les principes d'action pédagogique à différents niveaux. Sur le plan pratique, il est par exemple proposé d'« entrer en poésie contemporaine par l'intermédiaire d'une œuvre, d'un auteur, d'une démarche particulière » (on prend l'exemple de la métaphore chez Ponge comme instrument de connaissance du monde et de la langue); d'analyser les traitement de la référence; de témoigner d'une pratique en classe de troisième : l'étude d'une forme fixe, le sonnet, Queneau et Roubaud tenant le rôle d'initiateurs à la modernité et à l'écriture d'un « sonnet en prose ».

Au-delà de la référence à l'extrême contemporain et du souci revendiqué d'être actuellement moderne des deux numéros de la revue Le Français aujourd'hui ', on peut retenir deux propositions de Daniel Delas. Ce dernier, s'appuyant sur la notion de rythme, empruntée à Meschonnic ( $\left.\mathrm{n}^{\circ} 114\right)$, propose une approche multiple en esquissant l'évolution de la lecture du poème, puis en situant oralisation et écriture. Enfin, dans sa proposition d'«Un guide en poésie contemporaine, Du bref, du trop, du flux » ( $\left.\mathrm{n}^{\circ} 75\right)$, il pointe trois formes typiques de la modernité et des écritures singulières (Guillevic/Dupin, Réda/Stéfan, Venaille/Cliff).

Malgré les propositions des revues didactiques, la place des poètes contemporains est tout à fait lacunaire et non problématisée dans les programmes et les manuels, qui mettent en avant d'autres poèmes du xxe siècle. Quoi qu'il en soit, l'étude de la poésie ou des formes et genres poétiques continue de privilégier des groupement de textes concernant le surréalisme, la poésie et la résistance ou le thème plus ouvert de " poésie et résistance »; la répartition en séquences intègre par ailleurs d'autres époques (sonnet et lyrisme, poèmes en prose et formes libres, fonctions du poète et de la poésie, etc.).

Programmes et manuels : le fond et la forme

Le texte poétique contemporain n'est pas approché autrement que le texte de la Pléiade ou de la période romantique. Dans les Accompagnements des programmes, comme dans les revues ou les manuels, le groupement de textes permet au mieux par comparaison la mise en valeur des ruptures, autour des " traits spécifiques de l'écriture poétique " ${ }^{10}$ cadrés par les outils d'analyse que sont d'une part la typologie des textes (narratif, descriptif, dialogué), le lexique (champs lexicaux, champs sémantiques, registres de langue, connotation/dénotation), les modalités de la phrase et de l'énonciation ainsi que les temps verbaux, d'autre part la connaissance de certaines figures de rhétorique (images, comparaison, métaphore...) ainsi que les notions essentielles de rythme et de versification 
et le jeu sur les sonorités. Ainsi dans l'approche des formes poétiques, la norme est la métrique et la rythmique ; la séparation de la forme et du fond est de rigueur.

Deux exemples suffiront. Concluant une séquence intitulée "Sonnet et lyrisme» (Sabbah, 2001: 188), l'étude d'un poème d'Hier régnant désert suggère une approche contrastive autour de difficultés de réception du texte qui serait « liées à sa forme, à sa versification, aux mots qui la composent ", l'hypothèse de lecture suggérée étant que l'auteur " échappe aux différentes conventions qui conditionnent le thème et la forme "; mais c'est sans problématiser historiquement, ou dans la singularité d'une œuvre, ni la forme sonnet ni d'autres expressions du lyrisme, que la séquence aboutit à définir le registre lyrique (lexique, caractéristiques, "vie des êtres humains notamment lorsqu'il s'agit du lyrisme amoureux »). Pointant le fait qu'un texte en prose, L'huittre de Ponge, puisse être poétique en « instaurant un autre rapport au monde par le langage » et que l'écriture se soit dégagée des contraintes du vers régulier, le manuel (Aviérinos, 2000) propose dans un corpus rythmé et rimé l'étude du sonnet, forme majoritaire du corpus, ce qu'indique le programme officiel (de Louise Labé à Breton en passant par Nerval, le Parnasse et le Symbolisme).

Programmes et manuels : la prose contre le vers et l'approche limitée des « forme-sens »

Quand elle est approchée, la contestation du vers par la prose n'est d'ailleurs ni située historiquement ni théorisée clairement.

18 Ainsi, une séquence intitulée «Poèmes en prose et formes libres " (Sabbah, $2001:$ 196, 208, 372-273), dont le corpus s'étend de Gaspard de la nuit à des textes de Gracq et de Char, ne s'empare pas du thème de la modernité, définie par Baudelaire dans la lettre à Arsène Houssaye, pour marquer le sens d'une rupture introduite par la théorisation de la formesens dans l'histoire de la poésie lyrique (Meschonnic, 1993: 105-120). Troisième groupement de textes qui correspond à une troisième période de la poésie française, «Les tentations de la prose » réunissant Baudelaire, Rimbaud et Lautréamont constituent par contre l'un des traits caractéristiques de «l'épreuve de la modernité ». Dans un autre manuel (Rincé, 2001 :141, 146), on oppose à la modernité de la quadrette Baudelaire, Rimbaud, Mallarmé, Verlaine, les « écritures contemporaines » du xxe siècle, lequel est appréhendé selon deux axes répertoriant les « innovations ou remodelages du poème en tant que forme-sens et la multiplication des jeux sur le signifiant de la langue ». Le terme contemporain est alors défini par deux traits : la crise, puisque la poésie du xxe poursuit les perturbations formelles de la modernité, met en question les formes de la représentation, et la fête, caractérisée à la fois par la virtuosité, l'humour et par « une réaction joyeuse » [face à] la menace de « déconstruction » du genre poétique.

La notion est, on le voit, un fourre-tout qui superpose et simplifie des esthétiques et des poétiques hétérogènes et complexes. D'une part les « libérations formelles » juxtaposent l'Apollinaire des Poèmes à Lou et celui des Calligrammes, un texte de l'Espace du dedans, un poème en vers libre de Soupault et un poème en prose de Jaccottet, d'autre part les « jeux verbaux » font se rencontrer Le pain de Ponge, le gardien de phare de Prévert, un «bon dieu de bon dieu " quenellien de l'Instant fatal et le Sonnet lipogrammatique de Roubaud qui ouvre la Disparition de Perec. Il faudrait ici plus d'exigences théoriques: éviter de représenter l'évolution de la poésie comme une histoire linéaire devant aboutir à la situation actuelle alors que l'histoire de la poésie est conflit et multiplicité, ne plus simplifier tantôt vers la forme (le poème contemporain aurait des «audaces »), tantôt vers le sens («Poésie et présence au monde », "L'être dans les mots »). Même dans un usage didactique, le Sonnet lipogrammatique de Roubaud refait le texte mallarméen, le 
sonnet du «Cygne » comme le vocabulaire et la syntaxe mallarméens, la plume noire et la course du stylo sur la page remplaçant autant le « $\mathrm{e}$ » muet que le cygne de l'idéal. Comme l'intertextualité, la forme-sens chère à Meschonnic est un concept essentiel qui impose de considérer, plutôt que l'allusion biographique, le contexte du recueil et de l'œuvre pour entrer dans l'histoire d'un dire et d'un vivre. Au-delà des positionnements de circonstance ou des oppositions de surface, la lecture du contemporain a besoin de savoirs théoriques et pratiques.

Quels compromis pour la poésie contemporaine ?Un compromis théorique et pratique pour approcher le contemporain et l'« extrême contemporain "

Je n'ai trouvé qu'un ouvrage essayant d'intégrer à l'exigence scolaire le contemporain, sans se borner à la lecture méthodique ou à l'anthologie pour lire-écrire, Poésie contemporaine. 25 lectures et commentaires pour les lycées ", de Jean-François Massol (1995). Sont proposés dix-sept auteurs contemporains, qui ont publié entre 1980 et 1991, les uns légitimés dans les vingt dernières années par l'institution culturelle et l'institution scolaire : Guillevic, Jaccottet, Michaux, Norge, les autres reconnus dans le champ culturel et promus par Le Français aujourd'hui. Ils sont majoritairement publiés par l'éditeur de référence (Gallimard): Philippe Delaveau, Lorand Gaspar, Lionel Ray, Jacques Réda, Jacques Roubaud, Claude Roy, Jude Stefan, Henri Thomas; les éditions POL suivent avec Charles Juliet, puis Olivier Cadiot et Emmanuel Hocquard considérés comme écrivains d'avant-garde ou de rupture; édité par Messidor, Abdellatif Laâbi est le seul écrivain étranger en langue française ; James Sacré, déjà en cours de légitimation universitaire, représente un éditeur de province, Ryoan-Ji. On ne s'étonnera pas que cette entreprise novatrice concède à la légitimation éditoriale d'une part et se conforme par ailleurs aux nécessités didactiques : en dehors de l'extrait de l'Élégie $V$ de Hocquard et d'un extrait de Roméo et Juliette de Cadiot, en rupture avec les usages lyriques de l'école, l'épreuve du commentaire composé promeut Guillevic (extrait de Motifs), Michaux, Jaccottet (extrait de Beauregard), Réda (Retour au calme). Sans que les termes soient employés, d'un côté le lyrisme critique, de l'autre les tenants d'une nouvelle esthétique, que les auteurs définissent "sinon [comme] une théorie de la poésie, à tout le moins une réflexion amusée sur l'écriture » (c'est le Cadiot de L'Art poétic et des cut-up), ou situent dans une «modernité agressive et joyeuse» contre le pathos de la tradition lyrique française depuis Lamartine (Hocquard).

21 On ouvre sur la citation de Rimbaud («absolument moderne » - cf. note 2) pour évoquer la crise de la modernité et pour marquer, au-delà des contradictions, les «pratiques mêmes du palimpseste ", soit déférentes, soit post-modernes, des poètes choisis - Gaspar, Delaveau opposés à Hocquard et Cadiot. Quant à l'intermédiaire, Lionel Ray, son texte $\mathrm{Du}$ moderne est défini comme un parfait exemple de la mise en question de la post-modernité des années 1980 par un "lyrisme nouveau». On a ciblé un lectorat dont on connait l'habitus (professeur moderne informé faisant passer l'épreuve anticipée de français), en même temps qu'on utilise les commodités rhétoriques de l'exposé. On répartit les auteurs par thèmes : les expressions diverses de la passion, la poésie de la campagne et de la nature, la ville, la fuite du temps. Liée au poème en prose ou au vers libre, sinon à une écriture oralisée, la célébration des "petits riens, banalités, parfois merveille » réunit l'instant du lever du poète, moment de « sale vie mélangée à la mort » dans Quelque chose noir, l'humour d'une promenade à bicyclette au crépuscule de Réda (Retour au calme), les prosaïsmes d'un texte minimaliste d'Un après-midi à Marrakech, l'instant d'un «monde magnifié dans sa simplicité même» que constituent les «fumées d'étourneaux» d'A 
travers un verger. Certes, chaque partie propose une approche des textes conforme aux orientations des programmes officiels: lectures méthodiques, pistes de lecture (elles auraient parenté aujourd'hui avec la lecture cursive), commentaire composé ; chacune des orientations est articulée autour des cadres usuels de lecture du poème que l'on met en relation de manière variée: mise en page ou typographie, versification, syntaxe, système d'énonciation, temporalité, figures, champs lexicaux, cohésion et cohérence textuelle, rarement affirmée mais souvent utilisée la notion d'intertextualité. C'est une stylistique des écarts par rapport à la poésie lyrique qui définit les poètes de la rupture : mise en page avec phrases incomplètes constituant des paragraphes et repérages de la construction particulière que constituent «le texte troué et lacunaire» de Cadiot, «jeu magistral avec un texte ancien pour produire des effets de modernité » quand il s'agit de mettre en abyme chez Hocquard le travail et la quotidienneté du «je » narrateurtraducteur-professeur-lettré-voyageur-poète, lequel, reprenant Salluste, s'adresse à Catilina en fumant des Camel pour questionner le travail de la voix lyrique. Dans le courant de l'étude enfin, on trouve des références nombreuses à la totalité du poème ou du recueil; la tentative poétique de l'auteur est en principe située en fonction d'une appréciation qui réfère à l'ensemble de l'œuvre. Autant d'éléments qui dépassent le cadre du commentaire composé normalisé.

La poésie contemporaine entre institution culturelle et institution scolaire

Je m'en tiendrai à ce dernier exemple, même s'il reste encore à rendre compte des différentes pratiques de formation dans le cursus des professeurs des premier et second degrés, à considérer aussi le travail des séminaires ou d'ateliers de pratiques artistiques ainsi que l'articulation de l'action culturelle et de la formation des enseignants' ${ }^{12}$. Il y a là emblématiquement rassemblées les contradictions de l'institution culturelle et de l'institution scolaire :

23 - Il faut un minimum de théorisation qui situe à un moment donné les enjeux et les contradictions du micro-champ poétique, il faut encore une adéquation de cette analyse avec les réalités du système de réception (organisation de l'institution scolaire, pratiques de lecture ou/et d'écriture, discours critique...). La théorisation a aussi pour fonction de « moderniser » la lecture des œuvres passées : relire Rimbaud à partir de Ponge et sous le signe de la littéralité est un enjeu important ${ }^{13}$, mais ce type de tentative est rare, car limitée à quelques grands auteurs du programme; la collection "Textes et contextes " chez Magnard n'existe plus : elle serait très utile aux enseignants pour aborder le thème du programme « Ecrire, publier, lire».

24 - Il faut sans doute approcher le poème, le recueil et les œuvres autrement que par le commentaire: par une réception plus créative, plus ludique ou problématisante du contemporain. Le poème n'a de sens que dans l'œuvre, mais, sur le plan pratique, quand commencer la lecture d'un livre de poésie et de quel auteur (outils et pratiques diverses qui ne sont pas encore en usage) ? La formation universitaire à tous les niveaux préparet-elle à la réception du rap ou du slam, de la poésie sonore, du spatialisme, ou plus simplement à la mise en voix et en espace du poème ? S'il y a des ateliers d'écriture, qui sont les contemporains de référence? Quels sont les critères d'évaluation de l'écriture d'invention ou de l'épreuve d'invention au baccalauréat? Je renvoie aux accommodements envisagés par François Bon ${ }^{14}$. Bref, tout n'est pas possible!

25 - Le système de formation est nécessairement dans un compromis parce qu'il se réfère implicitement ou explicitement à des valeurs et à des discours, qu'il a devoir d'évaluer 
pour donner des certifications (ou des labels), qu'il y a la réalité des apprenants donc plusieurs «(il/)lisibilités » des œuvres. En témoignent les noms des poètes invités et les usages qui en sont faits à l'université comme dans les l'IUFM, et dans les établissements scolaires ; on n'oubliera pas ici la médiation parisienne du CNL. de la Maison des écrivains et du Printemps des Poètes.

- L'appropriation et la diffusion des œuvres obéissent à la loi du marché éditorial et du réseau culturel. Un bien culturel a une valeur symbolique : la poésie suscite lectures, articles, label et prix. Mais l'incidence n'est pas la même dans le système scolaire: le marché scolaire est ouvert à la poésie pour l'enfance et la jeunesse (à des collections), le marché universitaire privilégie le symbolique (la gratuité, la culture, être en pointe ou simplement informé) et l'usage théorique. Dans un lieu de formation d'enseignants, cette situation peut être gênante ou préoccupante.

Le système scolaire se préoccupe de " construire une culture » à partir de la littérature, de donner de celle-ci une vision historique et culturelle assimilable par le plus grand nombre. Faut-il alors privilégier les individualités poétiques? Quels écrivains contemporains - pour quelle exemplarité et pour quelle valeur? Dans la réalité, sans inscription sur les listes du baccalauréat ou si cette inscription reste sans suite (à l'oral), une œuvre et un écrivain ont peu de chance d'être reconnus, donc restent sans valeur légitimée. De plus, la distance critique et la reconnaissance dans le temps ne sont pas forcément une tare de l'enseignement : que ce soit la mode (Tel Quel il y a trente ans), la reconnaissance éditoriale ou anthologique à un moment donné, c'est la légitimation critique universitaire qui fonde la valeur actuelle d'une œuvre. Il y a certes des conflits entre les universitaires (chercheurs, critiques, poètes, professeurs), toujours est-il que la consécration de la poésie dans l'enseignement secondaire est l'inscription d'une œuvre dans la liste du bac en Terminale littéraire ${ }^{15}$.

C'est dans cette configuration que les termes « contemporain » et « modernité » prennent leur sens. C'est aussi par rapport à - avec, contre, sur - cette configuration didactique (le système pyramidal français passe de quelques essais créatifs au discours «sur » le poème - le commentaire composé) que l'introduction du contemporain et les pratiques nouvelles ont à trouver leur place.

\section{BIBLIOGRAPHIE}

Références critiques

BOURDIEU, P. (1992) : Les règles de l'art, Seuil.

Cahiers de Poésie-rencontres (Les), n 47-48, juillet 2001 (61 avenue Sidoine Apollinaire 69009 Lyon) : « Habiter en poète».

CEYSSON, P. (coordination), (2001), Guide pour aborder la littérature contemporaine au collège. L'exemple de Jean Joubert, CRDP de Lyon.

CHAILLOU, M. (collectif), (1990) : L'hexaméron. Il y a prose et prose, Le Seuil. 
DEBREUILLE, J.-Y. (1998) : Un poète dans la classe. Jean-Vincent Verdonnet, PUL., coll. IUFM, Lyon.

DELAVEAU, P. (1988) : La poésie française au tournant des années 80, Corti.

GLEIZE, J.M. (1993) : Arthur Rimbaud, Hachette/Université.

GLEIZE, J.-M. (1983) : Poésie et Figuration, Le Seuil.

GLEIZE, J-.M. (2001) : « Introduction à la poésie contemporaine » (à destination des bibliothécaires), sur :

<http://repertoiredepoesie.free.fr/presentation/poesie.html>

(au 29 oct. 2001).

LAUPIN, P. (2004) : Le courage des oiseaux. Une expérience d'écriture et de lecture avec des enfants en échec scolaire, Comp'act-Morari.

Littérature $\mathrm{n}^{\circ} 110$ (1998) : « De la poésie aujourd'hui. Chantiers, sentiers ».

Magazine littéraire, $\mathrm{n}^{\circ} 396$ (mars 2001) : « La nouvelle poésie française ».

MAULPOIX, J.-M. (1999) : Du lyrisme, Corti.

MESCHONNIC, H. (1993) : Modernité Modernité, Folio-essais nº 234.

PINSON, J.-C. (1995) : Habiter en poète, Champ Vallon, Recueil.

PINSON, J.-C. (2001) : Sentimentale et naïve. Nouveaux essais sur la poésie contemporaine, Champ Vallon, Recueil.

RICHARD, J.-P. (1964) : Onze études sur la poésie moderne, Le Seuil.

SITE de François Bon sur la littérature française contemporaine : www.remue.net, en particulier remue.net/ateliersd'ecriture.

Publications didactiques et pédagogiques

CNDP (1999) : Enseigner au collège. Programmes et accompagnement.

GEFFROY, M. (2000) : Poésie, du texte à l'image, CRDP de Grenoble/Delagrave, collection 1, 2, 3 ... Séquences.

L’École des lettres des collèges, 13, juin 2004. « L'écriture littéraire ».

L'École des lettres, II (second cycle), 8, 1999-2000.

Le Français aujourd'hui, 75, septembre 1986 : « Lisez-vous les contemporains ?».

Le Français aujourd'hui, 114, juin 1996 : «Il y a poésie \& poésie ».

Le Français aujourd'hui (1990) : Aimer/enseigner la poésie aujourd'hui, Syros.

MASSOL, J.-F. (1995) : Poésie contemporaine. 25 lectures et commentaires pour les lycées, CRDP de Grenoble.

NRP (La Nouvelle Revue pédagogique), 1, Nathan, septembre 1999 : « Lire la poésie contemporaine au collège $»$.

Pratiques, 93, mars 1997 : «Enseigner la poésie moderne ».

SITE éduscol (2005) : Accompagnement du programme de littérature. Classe terminale de la série littéraire.

Manuels consultés 
Collège

ACHARD, A.-M. (1999) : Grammaire et expression $3^{e}$, Hachette.

ACHARD, A.-M. (1999) : Littérature et expression $3^{e}$. Des méthodes pour lire et écrire, Hachette.

BESSON, J.-M. (2000) : Texto collège. Le français en séquences, Hachette.

BORÉ, C. (1998) : Lettres vives $4^{e}$. Lecture, langue, écriture, Hachette.

BRINDEJONC, M.-C. (2000) : Français $6^{e}$. Parler, lire, écrire, étude de la langue, Magnard.

BRINDEJONC, M.-C. (2001) : Français en séquences 5ème, Magnard.

CARMIGNANI, F. (2000) : Français $6^{\grave{e} e}$. A travers mots, langue, expression, Bordas

CARMIGNANI, F. (2001) : Français $5^{e}, 12$ séquences, Bordas.

COLMEZ, F. (1999) : L'art d'écrire, Larousse/Bordas

POTELET, H. (1998) Français $4^{e}$, Hatier.

POTELET, H. (1998) : Français $3^{e}$, Hatier.

Lycée

AVIÉRINOS, M. (2000) : Français seconde. Textes, genres, histoire, méthodes, Bordas.

AVIÉRINOS, M. (2001) : Français première, Bordas.

RINCÉ, D. (2001) : Français $1^{\text {re }}$. Textes, analyse littéraire et expression. Nathan, collection lectures écritures.

SABBAH, H. (2001) : Français première. Des textes aux séquences, Hatier.

\section{NOTES}

1. Je négligerai le terme avant-garde. Je réfère au mensuel Magazine littéraire $n^{\circ}$ 396, à la revue Le Français aujourd'hui n 75, et à Henri Meschonnic (1993) : Modernité Modernité. Les poètes-universitaires sont encore bien présents dans la revue Littérature $n^{\circ} 110$ : « De la poésie aujourd'hui. Chantiers, sentiers ".

2. Le titre du premier développement est inspiré par l'article « Il faut être absolument moderne » [ "Adieu », dernier poème d'Une saison en enfer], « un slogan en moins pour la modernité » (H. Meschonnic, op. cit.). Je reprends la conceptualisation de J.-C. Pinson (1995) : Habiter en poète, qui me permet de lire les œuvres de J. Joubert, P. Laupin, M. Porcu, J.-Cl. Pinson dans les Cahiers de Poésie-Rencontres n 48-49 (juin 2001).

3. Les références de ce paragraphe et du paragraphe suivant renvoient au Magazine littéraire, $\mathrm{n}^{\circ} 396$, successivement aux pages 20, 22-23, 23, 26-28, 40-42.

4. Enseigner au collège. Programmes et accompagnement (CNDP, 1999).

5. Pour les manuels consultés, voir la liste détaillée et les références en bibliographie. 6.M. Geffroy (2000), A.-M. Achard (1999), M. Aviérinos, Bordas (2001).

7. Considérés comme représentatifs, les quatre manuels du second cycle consultés (voir bibliographie) enregistrent Ponge à cinq reprises ( « L'huître », « Le pain », « L'œillet »), Bonnefoy et Jaccottet (2 fois)... Ponge figure dans l'Accompagnement des programmes (sept. 2001), ainsi que Bonnefoy pris comme exemple des poètes contemporains (p. 40, voir infra note 15$)$. 
8. Pratiques n 93 : «Enseigner la poésie moderne », p. 10, p. 20 (article de K. Canvat et G. Legros, « Enseigner la poésie moderne ? »). La poésie contemporaine est présentée avec un enthousiasme quasi hagiographique dans le dossier « Lire la poésie contemporaine au collège », NRP (La Nouvelle Revue pédagogique) n¹, Nathan, septembre 1999. Cadou, D. Pobel, Bielatovski, J. Charpentreau, J.-P. Siméon, Malineau, Prévert et... A. Finkielkraut (mots-valises) voisinent ensemble... avec l'Oulipo, Senghor, Guillevic, G. Jouanard, Ponge, Reverdy, G. Miron, Aragon, Eluard, Michaux, Jaccottet, du Bouchet, Emaz, etc., pour se répartir dans des groupements de textes adaptés à chaque classe (bestiaire, herbier, jeux de langage en sixième ; poésie engagée, poésie lyrique en troisième) et « conformes aux Instructions officielles ».

9. Le Français aujourd'hui $\mathrm{n}^{\circ} 75$. Le Français aujourd'hui $\mathrm{n}^{\circ} 114$ : «Il y a poésie \& poésie », dont le titre - clin d'œil pour initiés (référence à l'œuvre collective de Chaillou, Deguy, Delay, Michel, Roche, Roubaud, L'Hexaméron. Il y a prose et prose) - et le contenu affichent explicitement le souci d'être de circonstance et d'avant-garde. On n'oubliera pas dans cette revue d'association d'enseignants de français la chronique régulière et informée sur la poésie de T. Hordé, puis de D. Delas et S. Martin, ni Aimer/enseigner la poésie aujourd'hui (1990). De manière sommaire, je privilégie les traits saillants d'une approche contemporaine chez D. Delas.

10. Par exemple, dans la revue L'École des lettres, II (second cycle), 1999-2000, nº 8, un groupement de textes concernant les amours adolescentes, appréhendé dans une cohérence diachronique (poésie romantique, poésie du mouvement symboliste - du Rideau de ma voisine de Musset à Roman de Rimbaud), est utilisé pour définir « les traits spécifiques de l'écriture poétique » (tableau de synthèse : scène, sujet, espace-temps, énonciation, situation initiale/finale, personnages, statut du texte, tonalité) et se clôt sur le plan d'un commentaire composé de Vieille chanson du jeune temps (Hugo, Les Contemplations) en trois points : « le charme du récit, les personnages ou la rencontre impossible, le regard distancié ». Qui oserait suggérer les sonnets d' « Enfance » ou « Adolescence » dans Autobiographie de William Cliff (La Différence, 1993) ?

11. Le privilège accordé à cet ouvrage exemplaire me permet de ne pas dépouiller exhaustivement bon nombre de revues pédagogiques qui s'en s'ont inspirées. De plus, l'ouvrage est en continuité en tout avec le numéro 75 du Français aujourd'hui (cité p. II) ; il se réfère amplement à $P$. Delaveau (1988). Les citations et références qui suivent renvoient aux pages 32-33, 41-47, 3, 85-91, 180, 25-31.

12. Je renvoie pour d'autres pratiques (approches actives par création d'une anthologie, par réception ouverte de textes, créativité et, plus rarement, création...) à trois titres : Jean-Yves Debreuille (direction), Un poète dans la classe. Jean-Vincent Verdonnet, PUL, coll. IUFM, Lyon, 1998 ; Pierre Ceysson (coordination), Guide pour aborder la littérature contemporaine au collège. L'exemple de Jean Joubert, CRDP de Lyon, 2001 ; Patrick Laupin, Le courage des oiseaux. Une expérience d'écriture et de lecture avec des enfants en échec scolaire, Comp'Act - Morari 2004.

13. Voir par exemple J.-M. Gleize (1993) et J.-M. Maulpoix (1999).

14. Il envisage huit critères pour l'« évaluation sous forme de baccalauréat français »:1) richesse dans l'invention, le sensible, l'imaginaire ;2) audace de structure, affirmation formelle ; 3) qualités de chant et plastique de la langue, audace de syntaxe ; 4) résonance $\mathrm{du}$ monde, inscription du dehors ; 5) résonance intellectuelle et culturelle ;6) dialogue avec l'énoncé ou la contrainte ; 7) plaisir du texte ; et 8) « admissible pour l'examen, si cela ne l'est définitivement pas pour l'atelier de classe, maitrise de grammaire et orthographe ». Document disponible sur le site : <www.remue.net/atel/INV01bis.html>. 
Le didacticien de service repère la place considérable de la subjectivité dans bon nombre de critères et prévoit les difficultés de sa mise en place!

15. Depuis 2001, Nadja, de Breton, a représenté le registre poétique (domaine « Langage verbal et images - Littérature et langages de l'image »). Les Planches courbes (Mercure de France, 2001), d'Yves Bonnefoy, sera au programme en 2005-2006 (domaine « Littérature contemporaine »). Selon l'Accompagnement du programme de littérature (Site Eduscol : juin 2005), ce recueil constitue « un moment d'élucidation, d'éclaircissement des énigmes à partir desquelles ont été écrites un grand nombre d'œuvres poétiques depuis un siècle ». À l'usage des professeurs, est présentée « une problématique claire : faire advenir la Présence »; à l'usage des élèves, quatre entrées principales sont proposées : " variations sur des motifs simples ; intertextualité, mythes et figuration de l'entreprise poétique ; la conception de l'écriture de Bonnefoy ; le sujet de la poésie ». Bref, au sommet de la pyramide, la lecture du recueil et de l'œuvre s'articule avec les problématiques poétiques récentes.

\section{RÉSUMÉS}

A un moment où l'institution culturelle et l'institution scolaire expérimentent des dispositifs novateurs, les palmarès se multiplient, divers réseaux se constituent, fondés sur des enjeux et des positions engageant la conception même du «moderne » et du «contemporain ». Or, un bien culturel a une valeur symbolique dont l'incidence est différente selon le cursus scolaire. Quelles sont les « règles de l'art » qui régissent le passage du microchamp du poétique (éditeurs, revues, critiques) à la légitimation des œuvres dans l'institution (collège, lycée, université) ? En analysant les textes officiels, des revues, des manuels, on décrit les contradictions à l'œuvre dans la transposition didactique de la poésie contemporaine, laquelle ne peut être appréhendée que dans des compromis d'ordre théorique et d'ordre institutionnel, étant entendu que le baccalauréat littéraire et l'Université sont les clés du système.

\section{AUTEUR}

PIERRE CEYSSON

UFM de Lyon, Équipe Réel. 